\title{
Bragg diffraction from indium phosphide infilled fec silica colloidal crystals
}

\author{
H. Míguez, A. Blanco, F. Meseguer,* and C. López \\ Instituto de Ciencia de Materiales de Madrid, CSIC, Campus de Cantoblanco, E-28049 Madrid, Spain \\ and Unidad Asociada, CSIC-UPV, Departamento de Física Aplicada, Universidad Politécnica de Valencia, 46022 Valencia, Spain
}

H. M. Yates and M. E. Pemble

Department of Chemistry, University of Salford, Salford, Manchester M5 4WT, United Kingdom

V. Fornés and A. Mifsud

Instituto de Tecnología Química, CSIC-UPV, Avenida Los Naranjos s/n, 46022 Valencia, Spain

(Received 15 May 1998)

\begin{abstract}
Here we present results on Bragg diffraction in the optical range from indium phosphide infilled solid face-centered-cubic silica colloidal crystals. Scanning electron microscopy and optical properties indicate that the semiconductor is homogeneously grown within the three-dimensional lattice of voids in the colloidal crystal. The photonic-crystal behavior of the periodic dielectric is enhanced as a result of the high contrast of dielectric constants when InP is introduced.

[S0163-1829(98)02848-3]
\end{abstract}

Photonic crystals have attracted much attention since they were first proposed a decade ago. ${ }^{1,2}$ These materials exhibit a periodic spatial modulation of the dielectric constant, which is responsible for the appearance of diffraction effects in a region of frequencies determined by the distances in which the modulation occurs. ${ }^{3}$ Important technological applications have been proposed for such materials when designed to operate in the visible region of the spectrum. ${ }^{4}$ In that direction, a great attention has been paid recently to the photonic crystal behavior of colloidal crystals. ${ }^{5-11}$ These are made of submicrometric particles arranged in three-dimensional (3D) periodic structures in a medium of different dielectric constant, thus presenting Bragg diffraction effects in the optical region. However, in order to develop a technology based thereupon, some of their physical features have to be improved. A main point is that a dielectric constant contrast of $\varepsilon_{1} / \varepsilon_{2}$ $\approx 16$ is needed, ${ }^{12}$ rather than $\varepsilon_{1} / \varepsilon_{2} \approx 2$ that has been reached so far. Furthermore, a material with applications in photonics should be easy to handle, which will pose restrictions on its mechanical stability.

We have studied the photonic crystal properties of InP infilled mechanically stabilized face-centered-cubic (fcc) colloidal crystals made of silica spheres (opals). Using metalorganic chemical-vapor deposition (MOCVD), we have grown $\operatorname{InP}(\varepsilon \approx 12)$ within the void lattice of the silica matrix. The homogeneous distribution of the high $\varepsilon$ guest material in the low $\varepsilon$ host achieved by this method gives rise to enhanced photonic crystal properties as has been proved by optical reflectance measurements. Bragg reflections in the visible and near infrared (NIR) region caused by the crystalline planes in the $\mathrm{SiO}_{2}$-InP composites are between 1.5 and 2 times more intense than those presented by the bare silica matrix. Different sphere-size colloidal crystals have been infilled, hence a systematic study of the variation of the optical properties vs the sphere size was possible. It showed that the optical region in which the photonic behavior is observed can be accurately tuned by controlling the sphere size of the matrix.
Mechanically stabilized fcc silica colloidal crystals with the sphere diameter ranging from 200 to $550 \mathrm{~nm}$ were prepared following a recipe that has been previously described in detail. ${ }^{9,13}$ As a result of the preparation process, the spheres are in contact and, therefore, the lattice constant of the samples is determined by the radius of the particles. The fcc ordering and optical features of the host were checked by scanning electron microscopy ${ }^{14}$ (SEM) and optical transmission, respectively. ${ }^{10} \mathrm{InP}$ was formed in the host by atmospheric pressure MOCVD by a method similar to that used by the authors ${ }^{15}$ to infill opals from an alternative source. Growth conditions employed in this paper are the result of a systematic study performed to control the amount, quality, and distribution homogeneity of the InP introduced. ${ }^{16}$ Basically, there is a pregrowth heat treatment under hydrogen to remove any moisture present, followed by growth of the semiconductor. By changing the pregrowth temperature, the deposition temperature of reactants (trimethyl-indium and phosphine) and the number of cycles for each one, it is possible to control the amount of infill in the samples.

SEM images of cleaved edges of the samples were taken to study the morphology and distribution of the semiconductor grown inside the colloidal crystals. A JEOL 6300 microscope was used for this purpose. Figure 1(a) corresponds to an internal $\{111\}$ family surface of a silica colloidal crystal (sphere size $\phi=380 \mathrm{~nm}$ ) before the infill is grown. In Fig. 1(b) an image of an internal $\{111\}$ facet of the same sample infilled with InP is shown. In this sample, semiconductor crystals with an average size of $60 \mathrm{~nm}$ can be clearly seen on the spheres surface. The Bohr radius for InP is $90 \AA$, therefore, no quantum confinement effects of the electronic bands of infilled InP should be expected. SEM images demonstrate that the InP is very homogeneously distributed inside the matrix and, as a consequence, the 3D periodicity of the host is inherited by the guest material. Also, $\mathrm{x}$-ray diffraction and micro-Raman spectroscopy analysis ${ }^{16}$ show that InP is of good quality. 

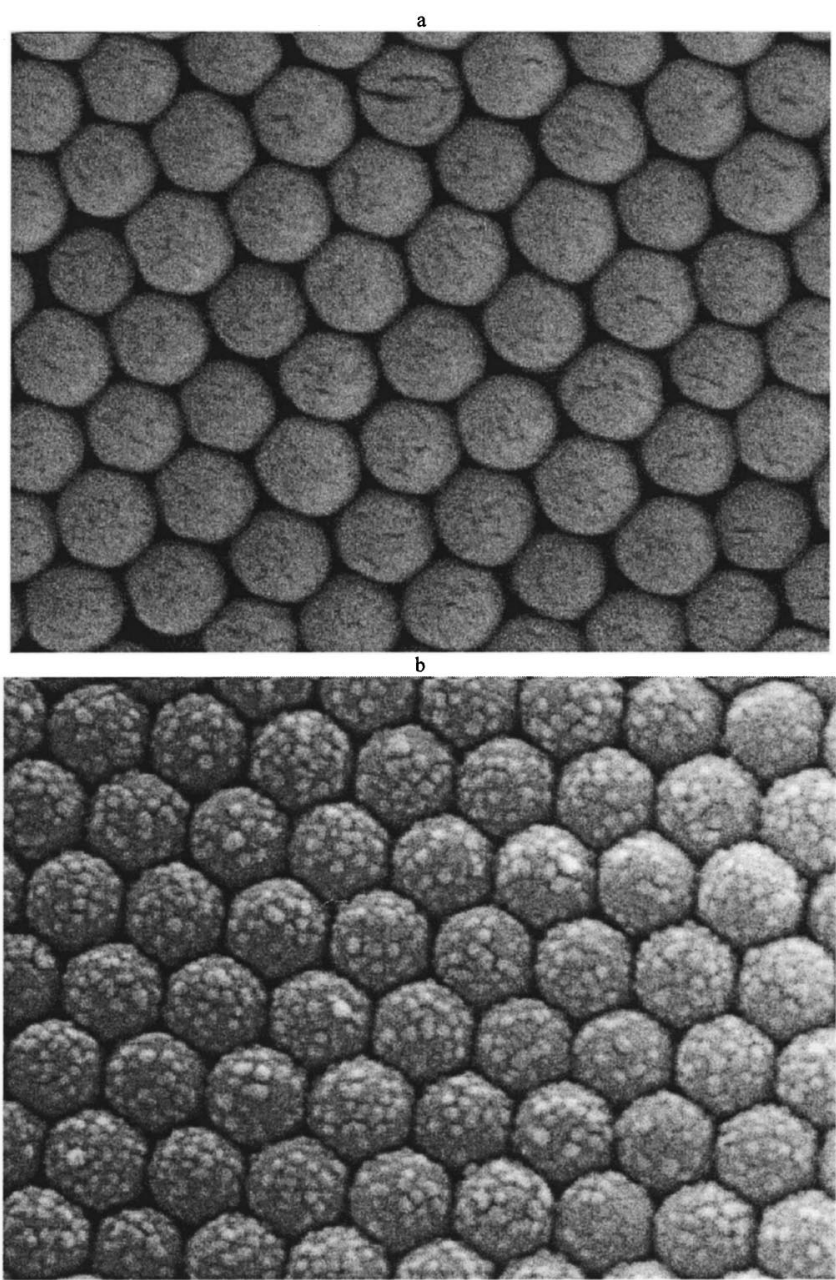

FIG. 1. SEM images of (a) internal (111) facet of a bare opal, and (b) a (111) internal facet of the same opal partially filled with InP. The formation of InP granules can be seen and their size estimated.

Photonic-crystal properties of bare and InP infilled crystals were studied using two different kinds of optical reflection measurements in the NIR and visible region. First, samples were shone with white light at different angles $\theta$ with respect to the (111) surface and the specular reflectance was measured. The geometry of the experiment determines

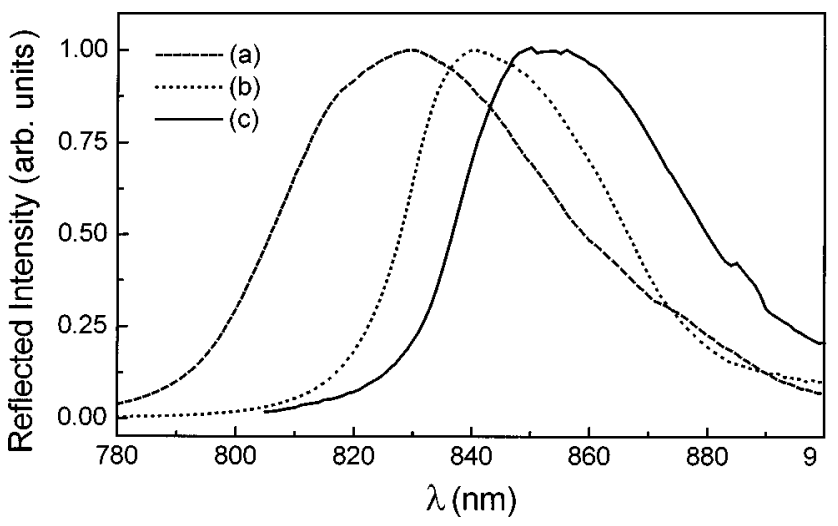

FIG. 2. Specular reflectance at $10^{\circ}$ for a bare opal (a) and the same opal loaded with different amounts of InP: $2 \%$ (b) and $4 \%$ (c) of the pore volume.

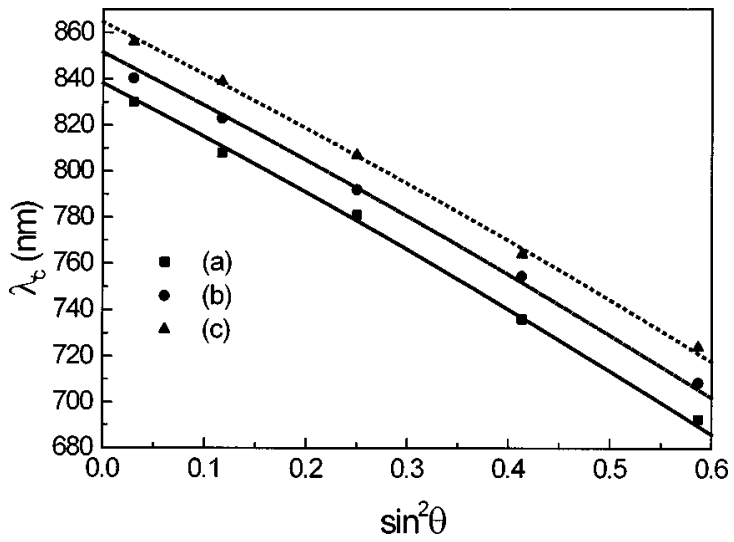

FIG. 3. Wavelength of maximum reflectance as a function of angle for the samples in Fig. 2 fitted to Bragg law. Data (a) correspond to the bare opal, (b) to the same opal with $2 \%$ of the pore filled with InP, and (c) to that with a $4 \%$ of the pore loaded. Lines are the different fits by using expressions (1) and (2).

that spectra contain information only about the (111) crystalline planes in the structure. Results for a bare colloidal crystal made of $380 \mathrm{~nm}$ spheres and the same sample loaded with different amounts of InP are shown in Fig. 2. There, normalized specular reflectance spectra at $\theta=10^{\circ}$ for the three samples are shown. The maximum observed in each case is caused by the coherent scattering from the (111) set of crystalline planes in the fcc structure. It can be observed that the position of the (111) Bragg reflection $\lambda_{c(111)}$ shifts towards higher wavelengths as the amount of InP infilled in the sample increases. In Fig. $3, \lambda_{c(111)}$ has been plotted against $\sin ^{2} \theta$ for the three samples: one bare opal and the same opal with two different loads. One can notice that as the opal is loaded the Bragg reflections occur at higher wavelengths for any angle. It should be seen also that, as Bragg reflections arise from the 3D order in the samples, the shift of the (111) optical band is only possible if a homogeneous distribution of the InP infilled exists.

In order to explain these optical characterization results, some general considerations are needed. ${ }^{17} \mathrm{~A}$ first approximation of $\lambda_{c(111)}$ can be obtained using Bragg law:

$$
\lambda_{c}=2 d_{(111)} \sqrt{\langle\varepsilon\rangle-\sin ^{2} \theta},
$$

in which $d_{(111)}$ is the distance between crystalline planes in the (111) direction, $\langle\varepsilon\rangle$ is the average dielectric constant of the composite, and $\theta$ is the angle between the incident radiation and the normal to the sample (111) surface. In addition, in our particular case, $\langle\varepsilon\rangle$ can be approximated by the expression:

$$
\langle\varepsilon\rangle=f \varepsilon_{\mathrm{SiO}_{2}}+f^{\prime} \varepsilon_{\mathrm{InP}}+f^{\prime \prime} \varepsilon_{\text {air }},
$$

$f, f^{\prime}$, and $f^{\prime \prime}$ being the filling ratios of silica $(f=0.74), \operatorname{InP}$ and air in the voids, respectively. $\left(f^{\prime}+f^{\prime \prime}=0.26\right.$. $)$ As can be readily derived from expression (1) an increase in either the average dielectric constant or sphere diameter causes an increase in $\lambda_{c(111)}$. Besides, it can be seen from expression (2) that InP infilled samples present higher average dielectric constant. Hence, it can be concluded that infilled samples should present Bragg reflections at higher wavelengths when compared with those coming from bare ones. Experimental 


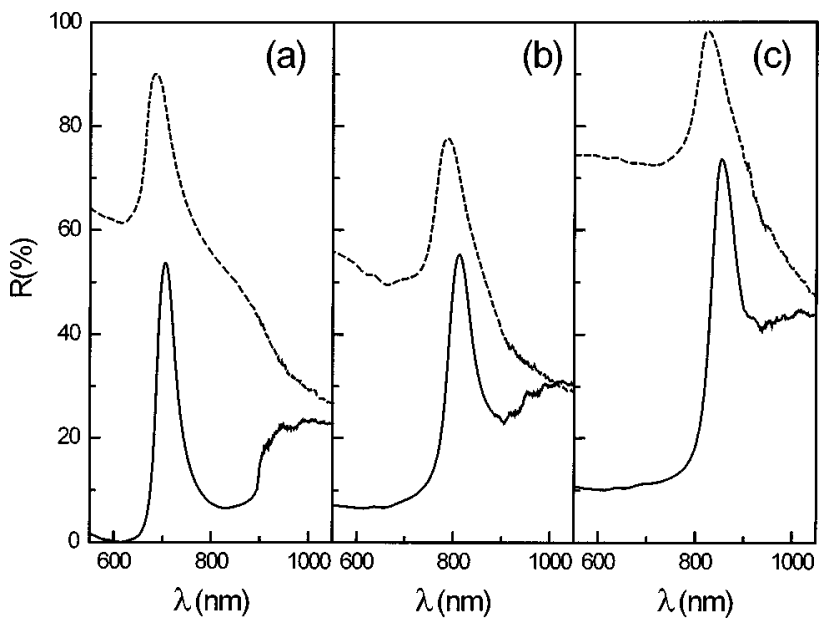

FIG. 4. Diffuse reflectance of the bare opal compared to the same opal after InP loading ( $4 \%$ of the pore volume). Three cases are shown for opals of increasing diameter: (a) $311 \mathrm{~nm}$, (b) $359 \mathrm{~nm}$, and (c) $380 \mathrm{~nm}$. As can be seen the Bragg peak can be tuned across the InP bulk band gap (at $900 \mathrm{~nm}$ ). Notice that these sort of measurements can only be performed in reflectance when the Bragg attenuation peak lies in the InP absorption range and, accordingly, attenuation by diffraction cannot be distinguished from ordinary optical absorption.

results shown here are in good agreement with these considerations. Lines in Fig. 3 correspond to fits of $\lambda_{c(111)}$ vs $\sin ^{2} \theta$ using expressions (1) and (2). ${ }^{18}$ The amount of InP infilled has been estimated from these fits, resulting in $2 \%(4 \%)$ of the pore volume for curve $b$ (curve $c$ ).

In order to obtain information on the efficiency of the photonic crystal effects observed in the samples, an integrating sphere accessory of a CARY5 spectrophotometer was employed to measure the diffuse reflectance. This configuration is not selective with respect to sets of planes as reflected light is collected irrespective of direction and reflectances are checked against a standard. In this case, samples were shone in at an angle of $\theta=3^{\circ}$ with respect to the (111) surface. In Fig. 4, integrated reflectance spectra for three different bare silica colloidal crystals (broken lines) and their InP infilled counterpart samples (solid lines) are shown. Samples whose results are shown in this figure were loaded employing the same growth conditions, InP content being around $4 \%$ of the pore volume in all three cases. Sphere sizes in each case are (a) $\phi=311 \mathrm{~nm}$, (b) $\phi=359 \mathrm{~nm}$, and (c) $\phi=380 \mathrm{~nm}$.

A few interesting features can be extracted from these spectra. First, it can be seen that by controlling the sphere size of the host, the photonic bands can be accurately tuned through the visible and IR region, as expected from expression (1) and, in particular, above and below the infilled InP band gap.

Second, a careful inspection of the reflectance peak linewidths systematically reveals that, relative widths, or gap to midgap ratios $\left(\delta \lambda / \lambda_{c}\right)$, are slightly larger in bare samples $(9 \%)$ than in the loaded structures (about $8 \%$ ).

Third, a very striking feature of the reflectance spectra is the spectacular reduction of the background diffuse reflectance in the samples with InP in the low wavelength range where the infill is absorbing.
Both line narrowing and background reduction can be explained in terms of the penetration depth for light in the InP gap. Beyond the absorption edge $(\lambda \lesssim 900 \mathrm{~nm})$ light is strongly absorbed and the size of the region probed is comparable to the domain size giving rise to natural linewidths in the reflectance peaks.

At $\lambda=688 \mathrm{~nm}$, for instance, the InP absorption ${ }^{19}$ index is $\kappa=0.27$, which means that the absorption coefficient is $\alpha$ $=2 \omega \kappa / c=4.9 \times 10^{-3} \mathrm{~nm}^{-1}$, which further means that the attenuation length is $1 / \alpha=200 \mathrm{~nm}$. If we assume only a $1 \%$ of the distance filled with InP ( $4 \%$ of the pore) the length in opal is equivalent to only $80 \mathrm{ML}$ of sample in Fig. 4(a). For sample in Fig. 4(b) it goes to $100 \mathrm{ML}$ and to $240 \mathrm{ML}$ in Fig. 4(c). What this produces is that light is actually probing only a very thin layer of the structures and the inhomogeneous broadening of the reflectance peak is negligible. When the opal is empty no absorption takes place and the buildup of a Bragg reflection is not restricted in the number of contributing monolayers allowing single-crystal domains from any depth to contribute and give rise to a wider peak. Furthermore, as the probed volume is reduced the incoherent scattering decreases which, in turn, lowers the background reflectance.

It is worth noting also that such thin structures are enough to produce Bragg reflection as the measured reflectance reaches $50 \%$. This leads to a very interesting result, namely, very small structure are able to develop photonic properties.

Finally, the intensities (percent reflectance above background) of the (111) Bragg reflections coming from the InP-SiO ${ }_{2}$ composites are between 1.5 and 2 times larger than those coming from their respective bare crystals. In order to explain this enhancement of the photonic crystal properties in InP infilled samples some further considerations are needed. It has been theoretically shown that a low infill of a high $\varepsilon$ material in a low $\varepsilon$ matrix causes a large increase in the scattering strength $\varepsilon_{r}$ of the periodic composite. $\varepsilon_{r}$, which can be thought of as the ratio scattering/no scattering of the structure, is defined by the expression derived by Sözüer, Haus, and Inguva, ${ }^{12}$

$$
\varepsilon_{r}=\sqrt{\left\langle\varepsilon^{2}\right\rangle /\langle\varepsilon\rangle^{2}-1}
$$

From expression (3) we obtain that, in a bare opal, $\varepsilon_{r}$ $=0.266$ while in a sample InP loaded up to a $4 \%$ of the pore volume, $\varepsilon_{r}=0.6$. The ratio of these scattering strengths is 2.25 , which is reasonably in agreement with the reflectance increase measured. Since InP has a very high dielectric constant $(\varepsilon \approx 12)$, even low InP loads yields a substantial increase of $\varepsilon_{r}$.

Therefore, optical experiments are in good agreement with the results derived from the theoretical considerations above indicated. It should be noticed that the change in the optical behavior observed indicates that the infilled material is $3 \mathrm{D}$ homogeneously distributed in the host.

In conclusion, we have observed Bragg diffraction from homogeneously InP infilled fcc silica colloidal crystals. Such periodic dielectrics present a high dielectric constant con- 
trast, which yields intense Bragg reflections in the visible and near infrared region. Analysis of the enhanced photonic crystal properties of InP infilled samples, when compared with bare ones, show a good agreement with theoretical predictions.
We would like to acknowledge M. Planes for his help during SEM characterization. This work has been partially financed by the Fundación Ramón Areces, and the Spanish projects CICyT MAT97-0698-C04 and GV-D-CN-08-12996.
*Author to whom correspondence should be addressed. Electronic address: fmese@fis.upv.es

${ }^{1}$ E. Yablonovitch, Phys. Rev. Lett. 58, 2059 (1987).

${ }^{2}$ S. John, Phys. Rev. Lett. 58, 2486 (1987).

${ }^{3}$ J. D. Joannopoulos, R. D. Meade, and J. N. Winn, Photonic Crystals (Princeton University Press, Princeton, NJ, 1995).

${ }^{4}$ J. D. Joannopoulos, P. R. Villeneuve, and S. Fan, Nature (London) 386, 143 (1997).

${ }^{5}$ W. L. Vos, R. Sprik, A. van Blaaderen, A. Imhof, A. Lagendijk, and G. H. Wegdam, Phys. Rev. B 53, 16231 (1996).

${ }^{6}$ I. I. Tarhan and G. H. Watson, Phys. Rev. Lett. 76, 315 (1996).

${ }^{7}$ J. M. Jethmalani and W. T. Ford, Chem. Mater. 8, 2138 (1996).

${ }^{8}$ Yu. A. Vlasov, V. N. Astratov, O. Z. Karimov, A. A. Kaplyanskii, V. N. Bogomolov, and A. V. Prokofiev, Phys. Rev. B 55, 13357 (1997).

${ }^{9}$ R. Mayoral, J. Requena, J. S. Moya, C. López, A. Cintas, H. Míguez, F. Meseguer, L. Vázquez, M. Holgado, and A. Blanco, Adv. Mater. 9, 257 (1997).

${ }^{10}$ H. Míguez, C. López, F. Meseguer, A. Blanco, L. Vázquez, R. Mayoral, M. Ocaña, A. Mifsud, and V. Fornés, Appl. Phys. Lett. 71, 1148 (1997).
${ }^{11}$ W. L. Vos, M. Megens, C. M. van Kats, and P. Bösecke, Langmuir 13, 6004 (1997).

${ }^{12}$ H. S. Sözüer, J. W. Haus, and R. Inguva, Phys. Rev. B 45, 13962 (1992).

${ }^{13}$ C. López, H. Míguez, L. Vázquez, F. Meseguer, R. Mayoral, and M. Ocaña, Superlattices Microstruct. 22, 399 (1997).

${ }^{14}$ H. Míguez, F. Meseguer, C. López, A. Mifsud, J. S. Moya, and L. Vázquez, Langmuir 13, 6009 (1997).

${ }^{15}$ H. M. Yates, M. E. Pemble, N. J. Johnson, S. G. Romanov, and C. M. Sotomayor Torres, J. Cryst. Growth 170, 611 (1997).

${ }^{16}$ H. M. Yates, M. E. Pemble, H. Míguez, A. Blanco, C. López, F. Meseguer, and L. Vázquez, J. Cryst. Growth (to be published).

${ }^{17}$ For a deeper theoretical analysis of the photonic structure of fcc colloidal crystals see V. Yannopapas, N. Stefanou, and A. Modinos, J. Phys.: Condens. Matter 9, 10261 (1997).

${ }^{18}$ Dielectric constant values in the visible and NIR region have been obtained from D. E. Aspnes and A. A. Studna, Phys. Rev. B 27, 985 (1983).

${ }^{19}$ O. J. Glembocki and H. Piller, in Handbook of Optical Constants of Solids, edited by E. D. Palik (Academic, Orlando, 1985). 